\title{
Chronic lung allograft pathology lesions in two rat strain combinations
}

\author{
Federica Pezzuto ${ }^{1}$, Francesca Lunardi ${ }^{1}$, Marta Vadori $^{2}$, Davide Zampieri ${ }^{1}$, Federica Casiraghi ${ }^{3}$, \\ Nadia Azzollini ${ }^{3}$, Stefania Edith Vuljan ${ }^{1}$, Marco Mammana ${ }^{1}$, Luca Vedovelli, ${ }^{1}$ Marco Schiavon ${ }^{1}$, \\ Dario Gregori, ${ }^{1}$ Emanuele Cozzi ${ }^{1}$, Federico Rea ${ }^{1}$, Fiorella Calabrese ${ }^{1}$
}

${ }^{1}$ Department of Cardiac, Thoracic, Vascular Sciences and Public Health, University of Padova Medical School, Padova, Italy; ${ }^{2}$ CORIT, Ospedale Giustinianeo, Padova, Italy; ${ }^{3}$ Mario Negri Institute for Pharmacological Research, Bergamo, Italy

Contributions: (I) Conception and design: F Calabrese, E Cozzi, F Rea; (II) Administrative support: None; (III) Provision of study materials or patients: M Schiavon, M Mammana; (IV) Collection and assembly of data: F Lunardi, SE Vuljan; (V) Data analysis and interpretation: D Gregori, L Vedovelli; (VI) Manuscript writing: All authors; (VII) Final approval of manuscript: All authors.

Correspondence to: Fiorella Calabrese, MD. Full Professor of Pathology, Department of Cardiac, Thoracic, Vascular Sciences and Public Health, University of Padova Medical School, Via A. Gabelli 61, 35121 Padova, Italy. Email: fiorella.calabrese@unipd.it.

\begin{abstract}
Background: Chronic lung allograft dysfunction remains an obstacle to long-term survival after lung transplantation. Two phenotypes have been described: obliterative bronchiolitis and restrictive allograft syndrome. Preclinical models are essential to analyze chronic lung allograft dysfunction pathophysiology.

Methods: Orthotopic lung transplants from 38 Lewis into Fischer 344 (Lew $\rightarrow$ F344) and 67 BrownNorway into Lewis $(\mathrm{BN} \rightarrow \mathrm{Lew})$ rats were performed in our center in the last decade. We carefully reviewed and quantified all grafts with chronic rejection (40 cases) (18 Lew $\rightarrow \mathrm{F} 344,22 \mathrm{BN} \rightarrow$ Lew) with the aim to investigate if histological changes of chronic lung allograft dysfunction could be also detected in rat grafts.

Results: All animals showed human reminiscent histological lesions. Early chronic rejection lesions were detected in $\mathrm{BN} \rightarrow$ Lew. End-stage chronic rejection with features of obliterative bronchiolitis was observed in $33 \%$ of Lew $\rightarrow \mathrm{F} 344$; end-stage with restrictive allograft syndrome chronic rejection in $67 \%$ and $80 \%$ of Lew $\rightarrow$ F344 and $\mathrm{BN} \rightarrow$ Lew, respectively. BN $\rightarrow$ Lew showed higher grades of endotheliitis, vascular fibrosis, and lower grades of lymphoid aggregates than Lew $\rightarrow \mathrm{F} 344$ ( $\mathrm{P}=0.007, \mathrm{P}=0.043, \mathrm{P}=0.004$, respectively).

Conclusions: Chronic rejection lesions in rat lung allografts mimic those in humans. The frequent occurrence of restrictive allograft syndrome-like lesions in $\mathrm{BN} \rightarrow$ Lew may be related to a higher degree of mismatch in this strain combination. These animal models could allow future mechanistic studies to better understand chronic lung allograft dysfunction pathogenesis.
\end{abstract}

Keywords: Lung transplantation; chronic rejection; animal model; rat model

Submitted Nov 30, 2020. Accepted for publication Feb 24, 2021.

doi: $10.21037 /$ jtd-20-3415

View this article at: http://dx.doi.org/10.21037/jtd-20-3415

\section{Introduction}

Transplantation is the only effective treatment for several end-stage lung diseases. Remarkable progress has been made in improving outcomes, although the current maximum median post-transplant survival is only 6 years (1). The main obstacle is represented by chronic lung allograft dysfunction (CLAD) which is observed in $50 \%$ of patients at 5 years post-transplantation (1).

CLAD has been recognized as a heterogeneous condition with about $50 \%$ of patients with an obstructive form [bronchiolitis obliterans syndrome (BOS)] and a smaller subset of patients with a restrictive pattern [restrictive allograft syndrome (RAS)] (2,3).

The pathology of BOS is sustained by the involvement of distal bronchioles, with a spectrum of lesions ranging 
from sub-epithelial peri-bronchiolar extracellular matrix deposition and fibrosis to lumen obliteration (3). In RAS the lesions are mainly located in the peripheral areas, with pleuro-parenchymal fibroelastosis (PPFE), diffuse alveolar damage (DAD), organizing pneumonia (OP), and scattered obliterative bronchiolitis (OB) lesions (4). A combination of pulmonary function test measurements, computed tomography scan, and less often histological findings contribute to CLAD phenotype classification $(3,5,6)$.

Although numerous risk factors have been identified, the specific pathogenetic mechanisms of CLAD still remain incompletely understood. The development of experimental models, mimicking the in vivo scenario, is of great importance to unravel the pathogenesis of CLAD, mainly with regard to the reproduction of RAS lesions. Several different experimental approaches have been developed to mimic the human aspects of lung transplantation. Currently, orthotopic lung transplantation in rodents is viewed as the surrogate model that more closely resembles lung transplantation in humans. There are numerous technical refinements that may improve the reproducibility of end point even if their use remains limited in the literature. In particular, only a limited number of rat models have been successfully used to reproduce OB aspects in orthotopic lung transplantations (7-9).

Over the last decade, several studies using rodent orthotopic lung transplantation have been carried out in our center with the purpose of examining the deep mechanisms of rejection and finding a model able to develop chronic allograft lesions. The present study is a careful histological revision of lung allografts from two different rat strain combinations transplanted in our center in the last decade. The principal aim was to investigate if all the histological changes reported as important in clinical CLAD could also be detected in orthotopic grafts in rats. The second aim of the study was to identify any potential difference between the two rat strain combinations by comparing the histological lung chronic rejection (CR) lesions observed in the two groups.

We present the following article in accordance with the ARRIVE reporting checklist (available at http://dx.doi. org/10.21037/jtd-20-3415).

\section{Methods}

We reviewed all rat lung grafts regarding these combinations in our center taking into consideration our new knowledge about human CLAD histological lesions, particularly those typically detected in BOS or RAS.

\section{Animal procedures and bistopathologic evaluation}

In the last decade 105 rats, 38 Lewis (Lew) into Fischer 344 (F344) (Lew $\rightarrow$ F344), 67 Brown-Norway (BN) into Lew $(\mathrm{BN} \rightarrow$ Lew), underwent left orthotopic lung transplants. Transplants were also performed using the Lew $\rightarrow$ Lew and F344 $\rightarrow$ F344 combinations (syngeneic transplants, used as control groups). All transplants of both groups were carried out by two surgeons, both expert in experimental surgery and microsurgery, following the techniques previously described $(7,8)$. After the transplantation procedures, rats of both groups received low dose of immunosuppression [cyclosporine A (CyA), $1.5 \mathrm{mg} / \mathrm{kg}$ s.c. from days 1 to 7] and antibiotics (ceftriaxone, $80 \mathrm{mg} / \mathrm{kg}$ s.c. from days 0 to 4 ). The choice of the low dose of immunosuppression was based on the large experience of our immunologist and on the successful development of post-transplant immunological complications obtained in previous experiments $(10,11)$. Animals were housed under standard environmental conditions, with a constant temperature $\left(22 \pm 1^{\circ} \mathrm{C}\right), 12$-hour light-dark cycle, with free access to food and water. Any recipient animal exhibiting signs of pain or distress that could not be timely alleviated with analgesics was humanely euthanized. Animals were euthanized at defined time points: 7, 15, 30, 60 and 90 days, respectively. At euthanasia, the entire heart-lung blocks were removed. The graft was measured, weighed, inflated, and subsequently fixed in formalin for 24 hours. Paraffin-embedded sections were routinely stained with hematoxylin and eosin, Masson trichrome, and elastic Verhoeff-van Gieson for morphological analysis. The histological examination followed the criteria of the International Society for Heart and Lung Transplantation (ISHLT) Lung Rejection Study (12), reporting, for each graft acute cellular rejection (ACR), lymphocytic bronchiolitis (LB) and OB. All relevant findings were also added to the final report. The results of the histologic evaluation were encoded in an electronic database in REDCap. The present work consists of a careful re-evaluation of all histological specimens by two dedicated expert lung pathologists (F Pezzuto, F Calabrese). The study focuses only on animals with CR features. Based on the current knowledge on CLAD phenotype lesions and according to a recently proposed grading system for mouse CR pathology (13), all histological lesions, including periairway, pleural, parenchymal and vascular fibrosis, airway obliteration, endotheliitis, lymphoid aggregates, epithelial 
hyperplasia, and flattening were quantified. Each parameter was scored in a semiquantitative manner, according to the grading system proposed by Martinu et al. (13) and categorized in low (score 0-2) and high (score 3-4) grades.

End-stage OB-CR was defined as a pathological alteration characterized by complete absence of the bronchiolar tract (the so-called vanishing bronchiolitis) associated with diffuse bronchiectasis and epithelial metaplasia of alveolar spaces; while an extensive pleural fibrosis and/or PPFE were seen in end-stage RAS-like CR. In the present study, we grouped three categories of euthanasia: "early" (within 7 days), "intermediate" (between 8 and 60 days), "late" (between 60 and 90 days). The occurrence of high-grade lesions was estimated for parenchymal fibrosis, pleural fibrosis, endotheliitis and vascular fibrosis taking into account the times of sacrifice.

\section{Statistical analysis}

Data were expressed as mean \pm standard deviation. Group comparisons were assessed with robust permutation tests: exact general independence test for ordered categorical and continuous variables and exact linear-by-linear association test for dichotomous variables using the $\{$ coin $\} \mathrm{R}$ package. Pathological parameters were also categorized following the times of euthanasia. Since in $\mathrm{BN} \rightarrow$ Lew combination no rat survived up to 90 days, the comparison was made only between "early" and "intermediate" timepoints for this group. Competing risk analysis was performed using the \{cmprsk\} $\mathrm{R}$ package to compare high-grade histological findings, taking into account the different times of euthanasia of each rat. R v.3.6.2 was used for the analysis (14).

\section{Ethical statement}

Experiments were performed under two project licenses (NO.: 3/2014: 244, NO.: project No. 9F5F5.49, authorized by Ministero della Salute, No. 17/2017-PR) granted by institutional ethics committee board of the University of Padova and Mario Negri Institute, respectively, in compliance with the "Principles of Laboratory Animal Care" formulated by the National Society for Medical Research and the "Guide for the Care and Use of Laboratory Animals", prepared by the Institute of Laboratory Animal Resources and published by the National Institutes of Health $7^{\text {th }}$ and $8^{\text {th }}$ editions. All procedures were conducted according to national (D.L. No. 26, March 4, 2014) and international laws and policies (EU Directive 86/609/EEC and 2010/63/EU) for the care and use of laboratory animals. The principles of replacement, refinement, reduction, aiming to conduct the research with animals in compliance with existing regulations and in the respect of the highest ethical standards, were fully respected.

\section{Results}

Eighteen lung recipients in the Lew $\rightarrow \mathrm{F} 344$ and 20 in the $\mathrm{BN} \rightarrow$ Lew combinations were euthanized within the 72 hours due to post-operative complications and/ or primary graft dysfunction. Forty rats $(60 \%$ of our case series) showed histological features compatible with CR, 18 belonging to Lew $\rightarrow \mathrm{F} 344$ model and 22 to $\mathrm{BN} \rightarrow$ Lew combination. No signs of CR were detected in the syngeneic transplants. CR lesions were observed in 5 (12.5\%), 24 (60.0\%) and $11(27.5 \%)$ rats euthanized within the first week, within 60 days and after 60 days, respectively. No rat of $\mathrm{BN} \rightarrow$ Lew combination reached the 90-day time point, being humanely euthanized within 60 days. In 7 cases with CR, ACR and/or LB coexisted (4 cases with A3B2, and 3 rats with A2B2, A2B1, A0B1, respectively).

\section{Histological assessment of CR associated lesions}

Lung parenchyma showed, in approximately half of the cases, a patchy distribution of the lesions. In 18 rats (45.0\%) end-stage lesions of CR were detected: 2 cases $(11.1 \%)$ were compatible with end-stage OB (Figure 1) while 16 of them (88.9\%) showed morphological changes compatible with end-stage RAS-like CR (Figure 2). End-stage OB-like CR was detected only in Lew $\rightarrow \mathrm{F} 344$ combination. End-stage RAS-like CR cases were present both in the Lew $\rightarrow \mathrm{F} 344$ (4 cases, $25.0 \%$ ) and $\mathrm{BN} \rightarrow$ Lew (12 cases, $75.0 \%$ ) combinations. In 2 of the $\mathrm{BN} \rightarrow$ Lew combination end-stage RAS-like CR, acute fibrinous and organizing pneumonia (AFOP) was also detected. Grafts with prevalent OB lesions were also characterized by several secondary changes such as intra-alveolar foamy macrophages, cholesterol clefts, and bronchiectasis. All the results are reported in Table 1 and Figure 3. A statistically significant difference between the Lew $\rightarrow \mathrm{F} 344$ and $\mathrm{BN} \rightarrow$ Lew strain combination scores was found for endotheliitis $(\mathrm{P}=0.007)$, vascular fibrosis $(\mathrm{P}=0.043)$ and lymphoid aggregates $(\mathrm{P}=0.004)$ (Figure $4 A$ ). Pleural fibrosis often showed features of PPFE. Interestingly, highgrade parenchymal fibrosis, pleural fibrosis, endotheliitis, and vascular fibrosis were mostly detected in $\mathrm{BN} \rightarrow$ Lew combination and appeared earlier compared to the 

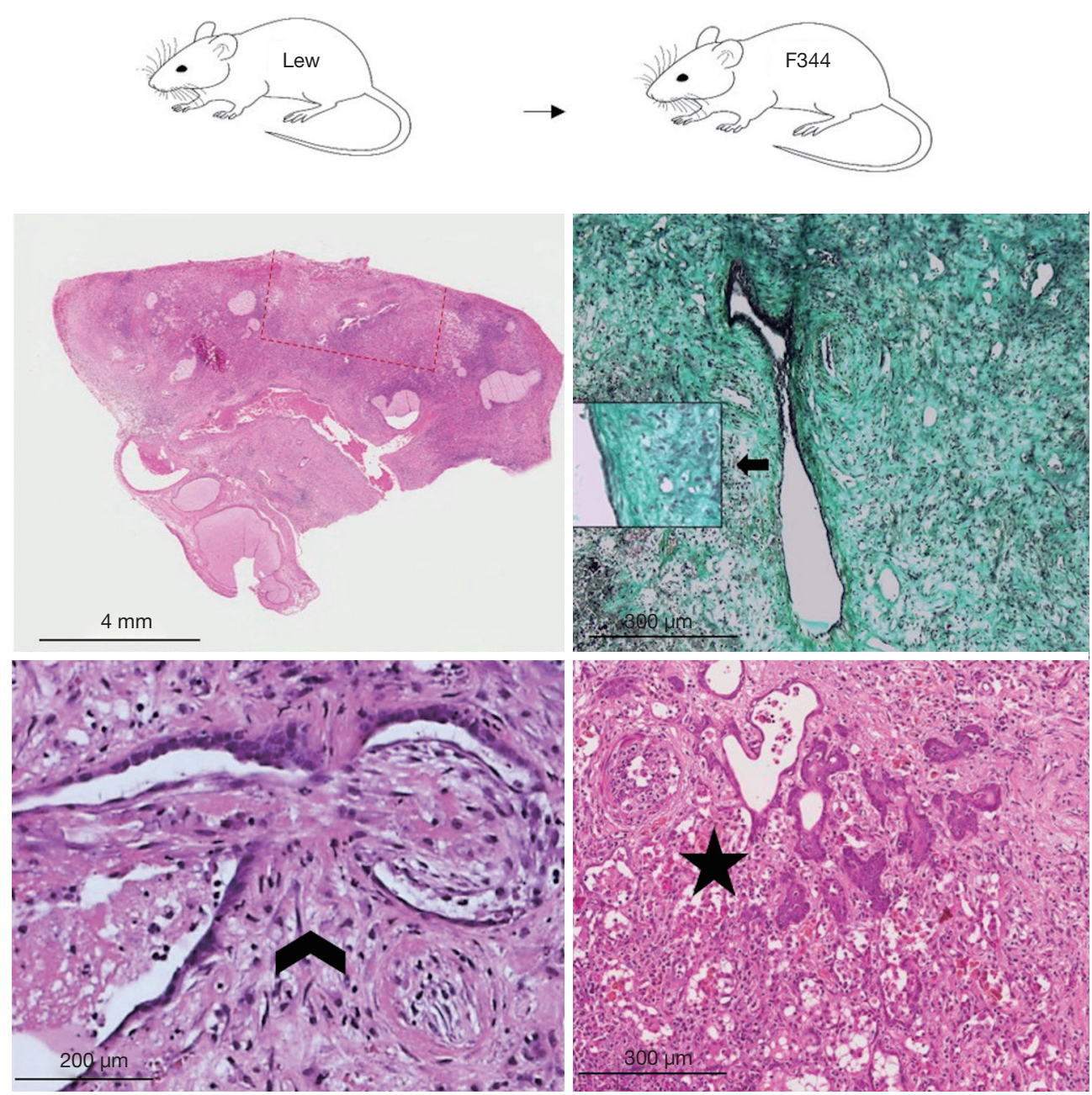

Figure 1 Representation of the Lewis into Fisher 344 model. The left transplanted lung shows diffuse bronchiolectases (panoramic view, scale bar: $4 \mathrm{~mm}$ ). At histology, some representative different features of severe obliterative bronchiolitis (arrow indicates the inset showing high magnification of epithelial flattening and significant fibrosis of sub-mucosal area-Masson trichrome stain, scale bar: $300 \mu$ m; arrowhead shows fibrotic plug in the lumen of obliterative bronchiolitis-hematoxylin and eosin stain, scale bar: $200 \mu \mathrm{m}$; star highlights bronchiolectases and squamous metaplasia of alveolar spaces-hematoxylin and eosin stain, scale bar: $300 \mu \mathrm{m})$.

Lew $\rightarrow$ F344 model, which showed these lesions after the first week (Figure 4B).

Finally, the results of competing risk analysis showed that the differences between the Lew $\rightarrow \mathrm{F} 344$ and the $\mathrm{BN} \rightarrow$ Lew combinations were statistically significant for parenchymal, pleural and vascular fibrosis $(\mathrm{P}=0.037, \mathrm{P}=0.019, \mathrm{P}=0.037$, respectively) (Figure $4 C$ ).

\section{Discussion}

In this study we reviewed 40 lung allografts with CR retrieved from two different rat strain combinations orthotopically transplanted in the last decade in our center. A thorough histological examination in agreement with a recently proposed scoring system for chronic lung allograft pathology (13) enabled the detection of lesions similar to those observed in humans with OB-like and RAS-like features.

As in humans, the distribution of bronchiolar remodeling with features of OB-CR lesions was often patchy. Indeed, OB lesions are frequently temporally and spatially heterogeneous and show a broad range of epithelial, fibrotic and inflammatory changes $(15,16)$. Differently from human lung allografts, OB-CR pathology of our rat grafts was more frequently characterized by the growth of fibrotic 

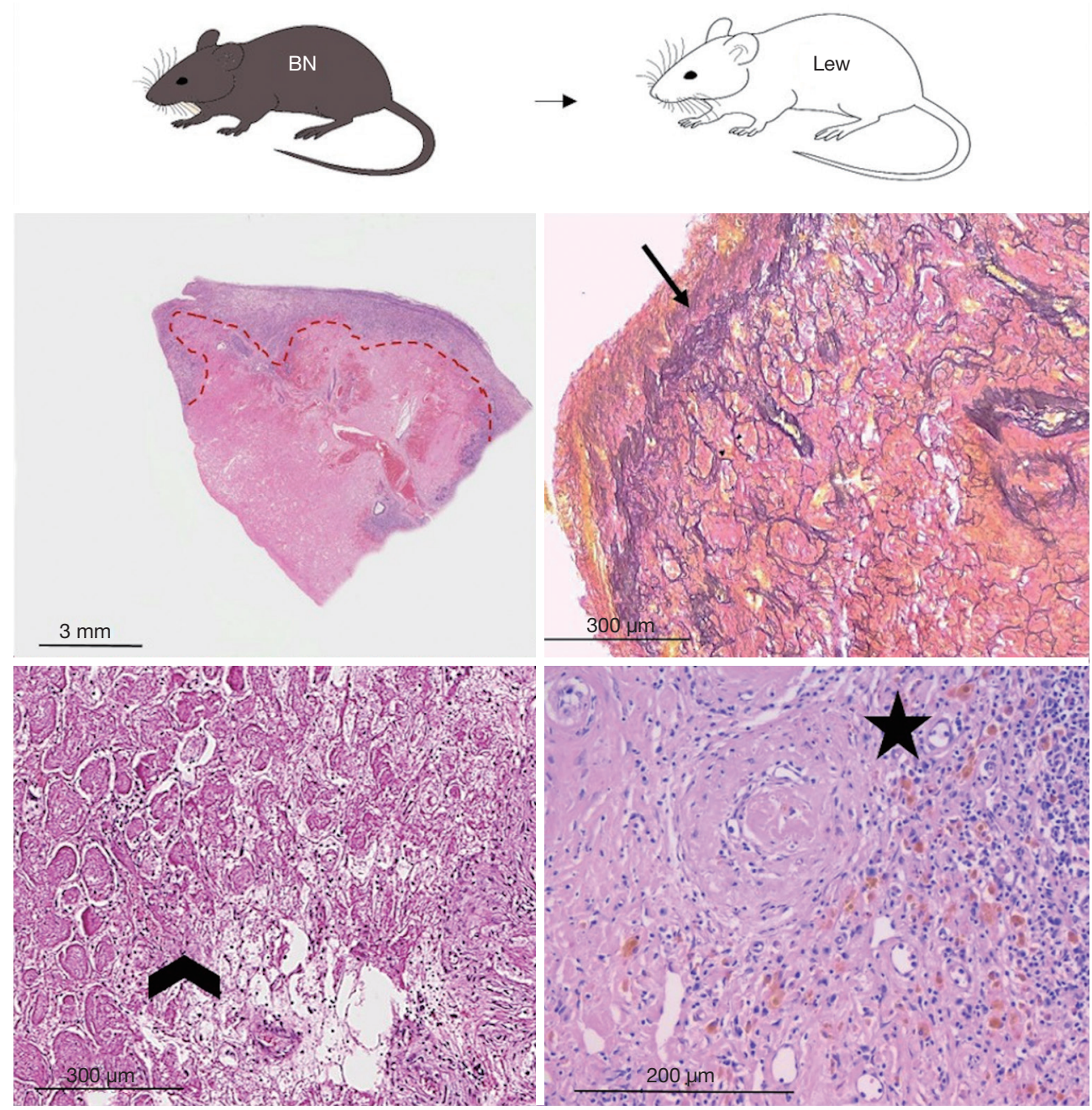

Figure 2 Representation of the Brown-Norway into Lewis model. The left lung shows pleural and subpleural fibrotic change with an important thickening of the visceral pleura (dotted line highlights pleural thickening — panoramic view, scale bar: 3 mm; arrow identifies pleuro-parenchymal fibro-elastosis_elastic Verhoeff-van Gieson, scale bar: $300 \mu \mathrm{m}$; arrowhead shows acute fibrinous and organizing pneumonia — hematoxylin and eosin stain, scale bar: $300 \mu \mathrm{m}$; star indicates marked vascular remodeling-hematoxylin and eosin stain, scale bar: $200 \mu \mathrm{m})$.

plugs into the lumen (obliterative features), instead of a concentric thickening and incomplete narrowing of the bronchiole (constrictive pattern). The pathogenetic reasons underlying such differences remain unclear (17).

In all grafts with OB-CR, particularly in more severe forms, several additional secondary changes were observed such as bronchiectasis, foamy alveolar macrophages or cholesterol clefts. These changes are often detected in clinical samples and could be considered in the routine evaluation of follow-up small transbronchial biopsies (TBBs), where adequate bronchiolar sections are often missing. In this context, it is noteworthy that a study from Stanford has previously reported a low sensitivity of TBBs in the detection of OB-CR lesions (up to $30 \%$ in a first report and $71.4 \%$ after the modification of the biopsy protocol) (18). The diagnostic yield of TBBs in the diagnosis of CR might be increased if the above reported secondary/additional changes would be taken into consideration. However, this aspect needs to be confirmed on further studies.

In our models with CR pathological lesions, peri-airway fibrosis was more evident than OB-CR. This finding could 
Table 1 Statistical description of the result analysis in all animals and in each group

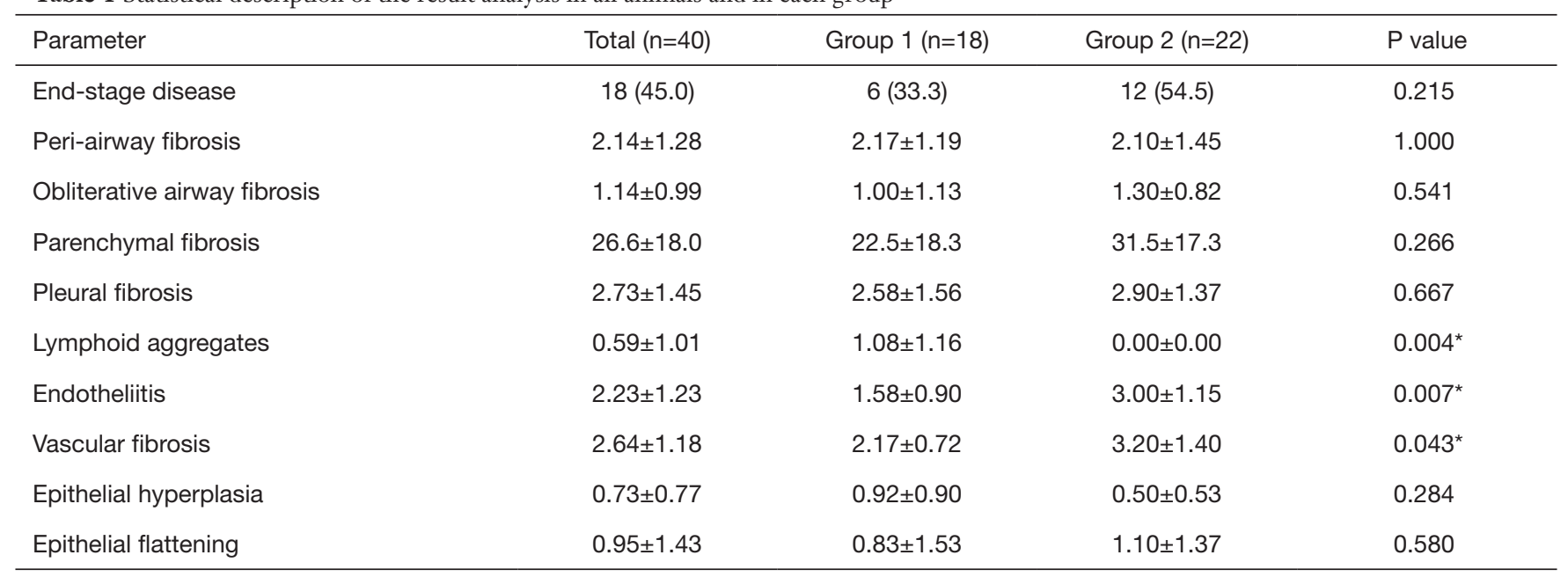

Data are expressed as number (\%) or mean \pm standard deviation. *, $\mathrm{P} \leq 0.05$.

reflect a better representation of large airways in tissue samplings. However, it is noteworthy to point out that large airways were significantly affected in the CR of our groups with a mean score greater than 2. Large airways are not considered in the current rejection grading system of clinical samples, even though several authors have more recently highlighted the need to reconsider the role of large airways $(19,20)$.

The two rat strain combinations used in our study showed several significant differences: end-stage OB-CR lesions were only detected in Lew $\rightarrow \mathrm{F} 344$ combination while end-stage RAS-like CR alterations were more evident in $\mathrm{BN} \rightarrow$ Lew model which also showed a significantly more prominent vascular remodeling, either as vascular fibrosis or endotheliitis. Vascular inflammation and fibro-intimal thickening are frequently detected in CLAD patients, especially in the RAS phenotype (21). Microvascular damage has been described adjacent to fibroelastosis in human RAS $(22,23)$. As suggested by some authors $(24,25)$, the presence of donor-specific antibodies and antibody-mediated rejection could be considered a risk factor for the onset of RAS. Further support is provided by the evidence that HLA-DRB1/3/4/5+DQA/B eplet mismatch was specifically associated only with RAS, but not with $\mathrm{OB}(26,27)$.

The presence of AFOP in only 3 cases of $\mathrm{BN} \rightarrow$ Lew combination provides additional data supporting our hypothesis that this strain combination is more susceptible to the development of RAS-like CR. AFOP is characterized by abundant deposition of fibrin in the alveolar spaces (e.g., fibrin ball), associated with mild interstitial inflammation.
This lesion suggests the possible contribution of several risk factors including infections, drugs and immune status. In the absence of such factors, it is considered idiopathic and is reported by several authors as a transient pathological process that can often lead to RAS (28).

The role of a humoral response in promoting fibrosis has also been suggested by Watanabe $e t$ al. in a mouse model of orthotopic lung transplantation. Indeed, in their studies the authors could show that, following ischemia-reperfusion injury, a B cell-dependent pathway was able to drive chronic lung allograft rejection with prominent parenchymal fibrosis and lymphoid aggregates. This process was associated with the presence of circulating autoantibodies and could be attenuated by B cell depletion (29).

Another statistically significant difference between the Lew $\rightarrow \mathrm{F} 344$ and the $\mathrm{BN} \rightarrow$ Lew strain combination was the presence of lymphoid aggregates, more prominent in Lew $\rightarrow \mathrm{F} 344$ model. The presence of lymphoid tissue in CLAD is still debated. It was first considered to be an immune mediated event, precursor of OB (30). Sato et al. described the occurrence of lymphoid neogenesis in CLAD, in relation to stromal resident cell activation (31). This finding was observed in all compartments of the lungs examined, thus suggesting the contribution of the inflammatory microenvironment in CLAD onset. Recent data (32) have reported a more pronounced structural organization of lymphoid follicles in RAS. As no conclusive results have yet been achieved, this topic remains an important point that needs to be deeply investigated.

We further compared all pathological findings, according 
A

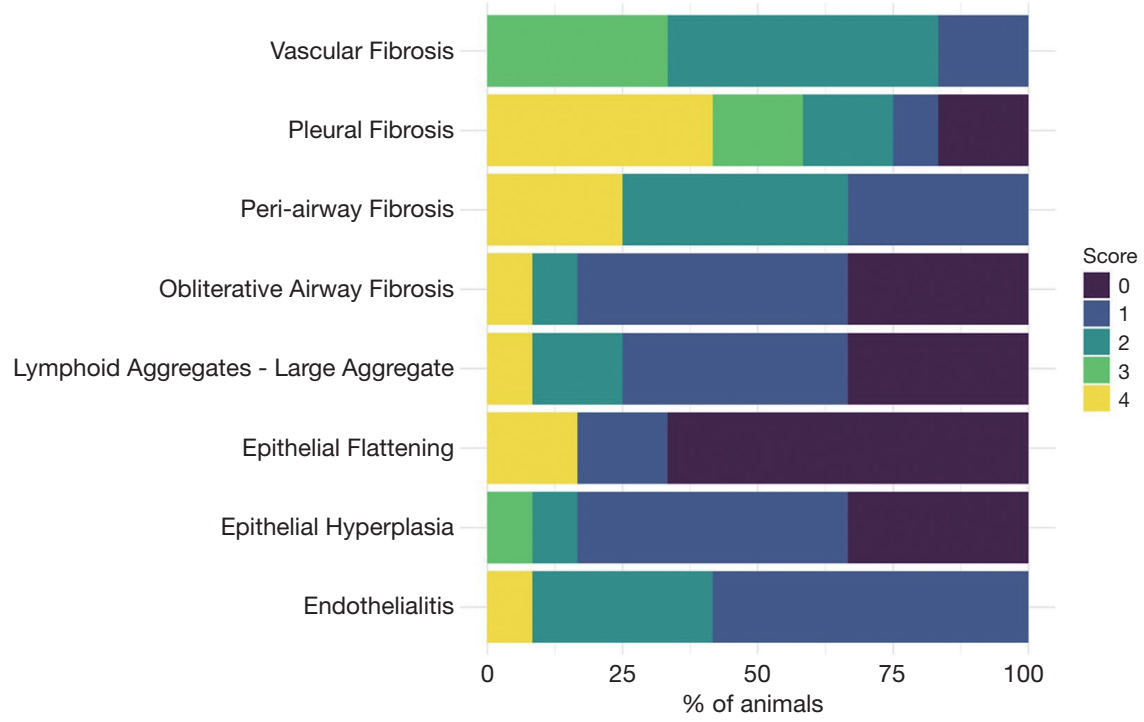

B

Brown-Norway into Lewis

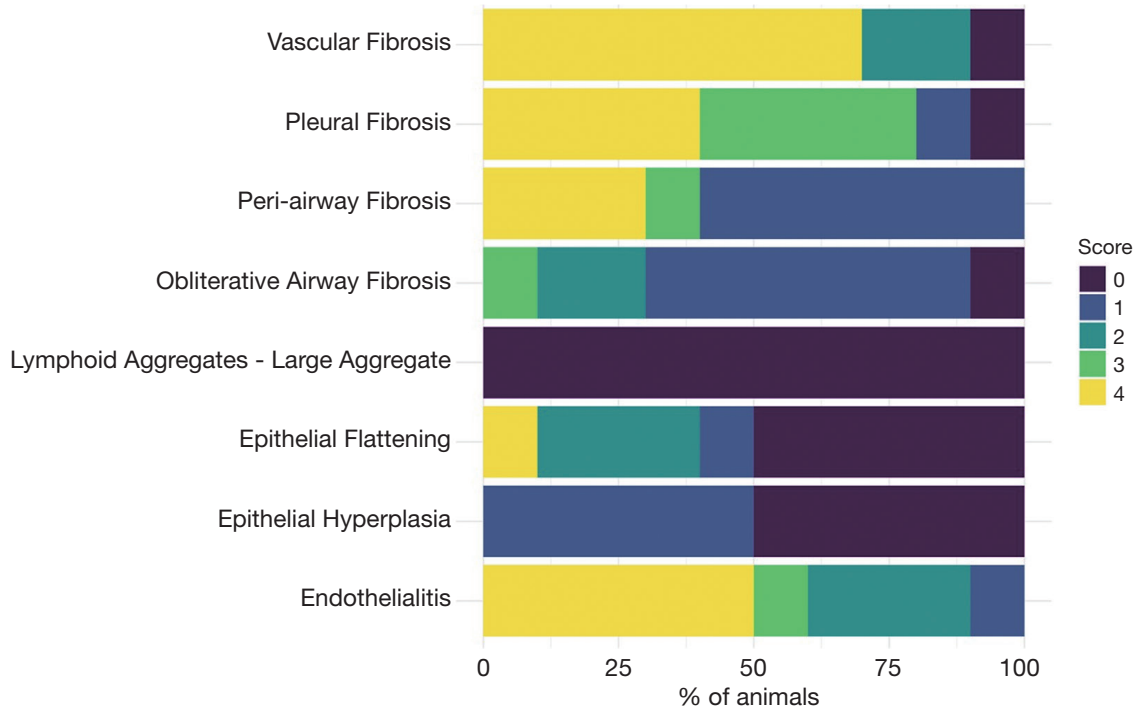

Figure 3 Distribution of scores in the Lew $\rightarrow$ F344 (A) and the BN $\rightarrow$ Lew (B) combinations. The graph shows the percentage of animals with each grade for all histological parameters.

to euthanasia. We detected pleural remodeling in both the Lew $\rightarrow$ F344 and the $\mathrm{BN} \rightarrow$ Lew model. However, highgrade pleural fibrosis and PPFE were detected earlier in the $\mathrm{BN} \rightarrow$ Lew than in the Lew $\rightarrow \mathrm{F} 344$ transplants. These findings lead us to speculate that in Lew $\rightarrow \mathrm{F} 344$ combination the pleural thickening could be related to other injuries. In $\mathrm{BN} \rightarrow$ Lew model the early occurrence of pleural fibrosis/PPFE combined with other lesions, such as endotheliitis and vascular fibrosis, support the hypothesis of a different etiology more likely related to immunological insults and may justify why no $\mathrm{BN} \rightarrow$ Lew transplants survived for a period longer than 60 days. This aspect seems to be in line with the clinical setting in which RAS is associated with a worse prognosis compared to OB (33). Even if the histology showed some characteristics of $\mathrm{CR}$, the event occurred at a very early time point, within the first week after transplantation. Early lesions of CR (within the first week after transplantation) have also 
been reported in other animal models (13). Although the majority of lesions was observed later on, at 14-28 days, some parameters such as peri-airway fibrosis and pleural fibrosis were already detected at 5-7 days. A plausible explanation for the earlier and more frequent RAS-like $\mathrm{CR}$ lesions in $\mathrm{BN} \rightarrow$ Lew combination could be related to a greater degree of mismatch of the minor and major histocompatibility complex loci in the $\mathrm{BN} \rightarrow$ Lew than in the Lew $\rightarrow \mathrm{F} 344$ model. This is also what has been observed in the clinical setting where the early development of CLAD occurs primarily in the presence of donor-specific antibodies (34) that accordingly, are related to HLA
A Endotheliitis

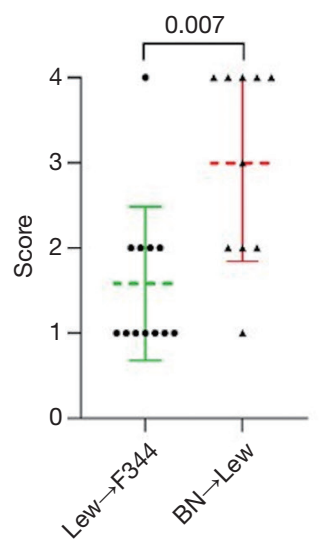

B

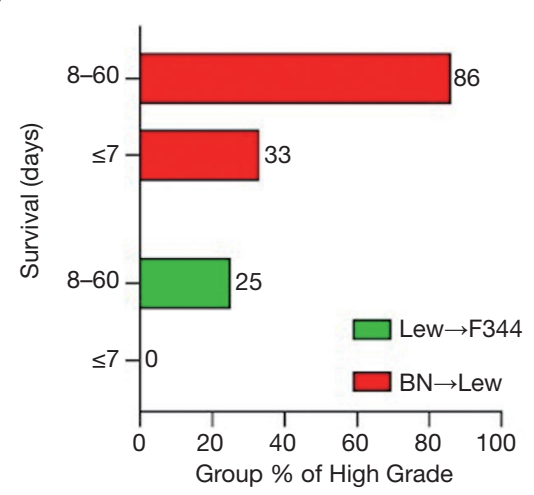

Endotheliitis

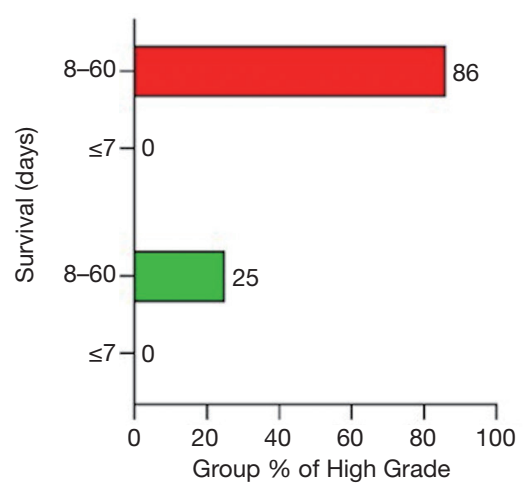

Vascular fibrosis

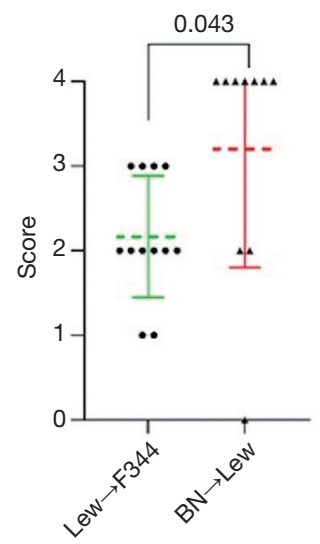

Lymphoid aggregates

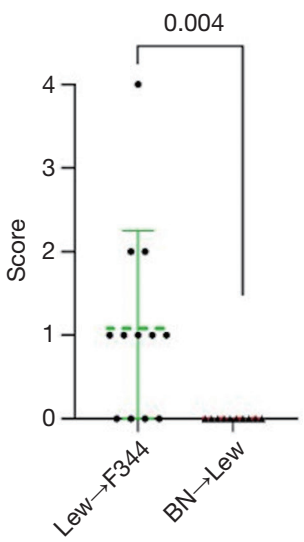

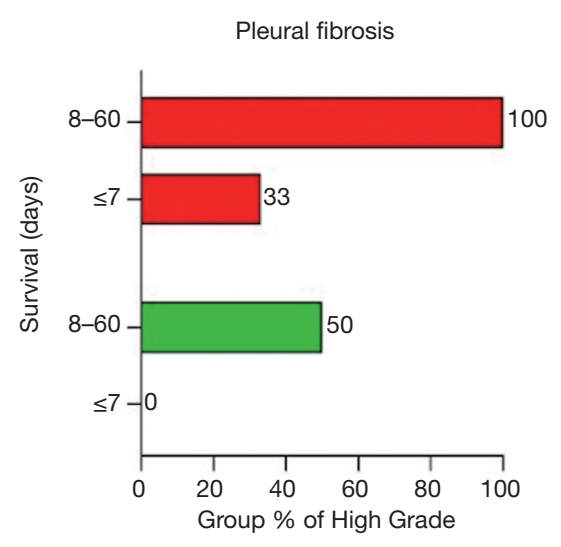

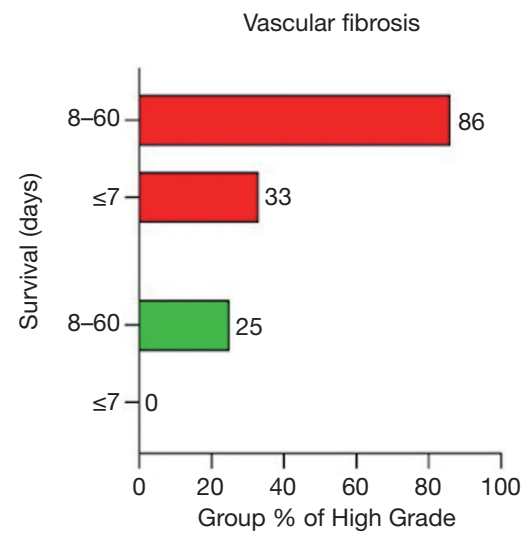




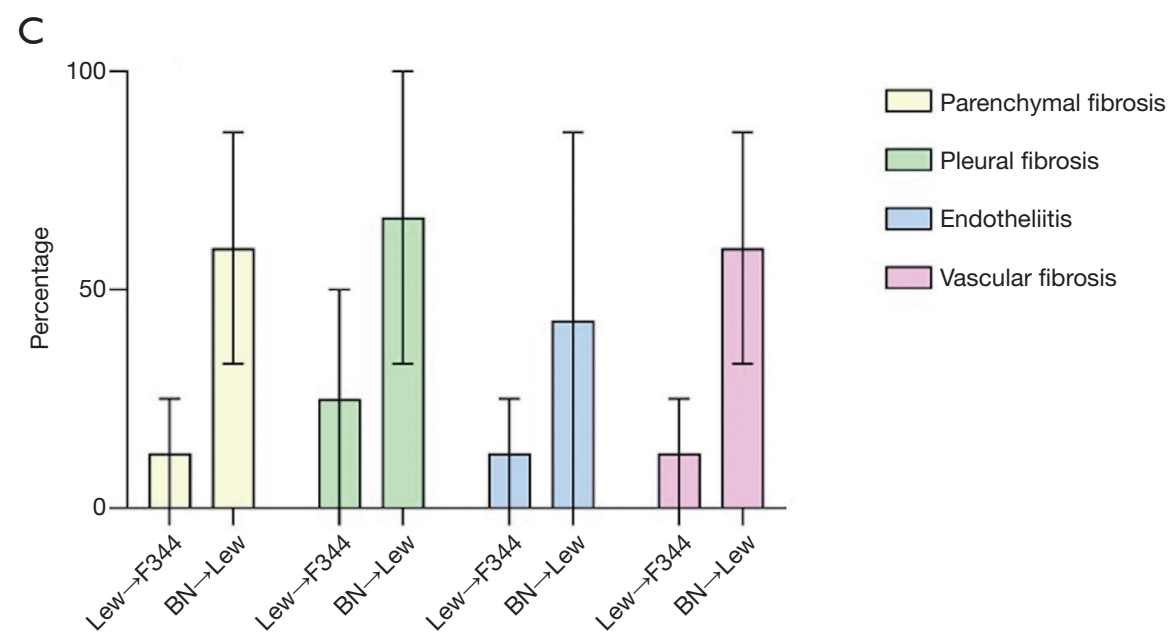

Figure 4 Comparison of the histological lesions between the two rat strain combinations. (A) Comparison of endotheliitis, vascular fibrosis and lymphoid aggregates between the two groups. Endotheliitis and vascular fibrosis were more prominent in $\mathrm{BN} \rightarrow \mathrm{Lew}$ combination while lymphoid aggregates were only detected in Lew $\rightarrow$ F344 model. Rats showing end-stage lesions were not scored. (B) Comparison of highgrade lesion percentages distinguishing early and intermediate timepoints. Although no statistical significance was achieved, high-grade parenchymal fibrosis, pleural fibrosis, endotheliitis, and vascular fibrosis were more evident and occurred at an earlier stage in $\mathrm{BN} \rightarrow \mathrm{Lew}$ transplants. (C) Competing risk analysis. Parenchymal fibrosis, pleural fibrosis, and vascular fibrosis were significantly higher in the $\mathrm{BN} \rightarrow$ Lew than in Lew $\rightarrow \mathrm{F} 344$ strain combination.

mismatches (35). Moreover, although our model is a single lung transplant model, the contralateral native lung may not have adequately compensated for the lost function of the transplanted organ, thus explaining the shorter survival of this rat strain combination. Indeed, in all rats and especially the $\mathrm{BN} \rightarrow$ Lew combination, we frequently observed an architectural remodeling in the contralateral lung with features of atelectasis (especially in graft with end-stage CR) or inflammation and/or interstitial fibrosis. While at this stage we do not have mechanistic explanations, it may well be that different factors such as mechanical (e.g., mechanical ventilation) (36) or immunological (e.g., antibodies against self-antigens) (37) events can impact on the contralateral native lung that at that stage can no longer be considered healthy.

Our study presents several limitations. First, evaluation of lung graft function in the rats remains a serious challenge. Second, the study was based on a retrospective analysis of grafts transplanted in our centre during the last decade, belonging to two distinct research programs. However, the therapeutic approach was similar and only two skilled surgeons performed all experiments, thus limiting the intraoperative variability. Micro-CT scan which could significantly help for the evaluation of graft dysfunction has only been recently introduced in our centre and will be available for future experiments.

\section{Conclusions}

In conclusion, Lew $\rightarrow \mathrm{F} 344$ and $\mathrm{BN} \rightarrow$ Lew combinations represent two orthotopic lung transplant rat strain combinations that show allograft pathological lesions similar to the alterations detected in human CLAD. $\mathrm{BN} \rightarrow$ Lew model showed more frequently lesions suggestive of RAS-like CR, a finding that could be related to a higher degree of immunological mismatch in this strain combination. The recently published grading system, evaluating histological lesions in mouse transplants, appears extremely useful in the study of CR pathological lesions, leading to the identification of crucial differences between rat experimental model strains. Should our findings be confirmed by further experimental studies, these two rat strain combinations could represent valuable animal models for better understanding CLAD pathogenetic mechanisms and possibly help identify appropriate treatment strategies.

\section{Acknowledgments}

The authors thank Dr. Judith Wilson for English revision. Funding: None. 


\section{Footnote}

Reporting Checklist: The authors have completed the ARRIVE reporting checklist. Available at http://dx.doi. org/10.21037/jtd-20-3415

Data Sharing Statement: Available at http://dx.doi. org/10.21037/jtd-20-3415

Conflicts of Interest: All authors have completed the ICMJE uniform disclosure form (available at http://dx.doi. org/10.21037/jtd-20-3415). The authors have no conflicts of interest to declare.

Ethical Statement: The authors are accountable for all aspects of the work in ensuring that questions related to the accuracy or integrity of any part of the work are appropriately investigated and resolved. Experiments and was performed under two project licenses (NO.: 3/2014: 244, NO.: project No. 9F5F5.49, authorized by Ministero della Salute, No. 17/2017-PR) granted by institutional ethics committee board of the University of Padova and Mario Negri Institute, respectively, in compliance with the "Principles of Laboratory Animal Care" formulated by the National Society for Medical Research and the "Guide for the Care and Use of Laboratory Animals", prepared by the Institute of Laboratory Animal Resources and published by the National Institutes of Health $7^{\text {th }}$ and $8^{\text {th }}$ editions. All procedures were conducted according to national (D.L. No. 26, March 4, 2014) and international laws and policies (EU Directive 86/609/EEC and 2010/63/EU) for the care and use of laboratory animals. The Principles of replacement, refinement, reduction, aiming to conduct the research with animals in compliance with existing regulations and in the respect of the highest ethical standards, were fully respected.

Open Access Statement: This is an Open Access article distributed in accordance with the Creative Commons Attribution-NonCommercial-NoDerivs 4.0 International License (CC BY-NC-ND 4.0), which permits the noncommercial replication and distribution of the article with the strict proviso that no changes or edits are made and the original work is properly cited (including links to both the formal publication through the relevant DOI and the license). See: https://creativecommons.org/licenses/by-nc-nd/4.0/.

\section{References}

1. Chambers DC, Yusen RD, Cherikh WS, et al. The registry of the International Society for Heart and Lung Transplantation: thirty-fourth adult lung and heartlung transplantation report-2017; focus theme: allograft ischemic time. International Society for Heart and Lung Transplantation. J Heart Lung Transplant 2017;36:1047-59.

2. Verleden GM, Raghu G, Meyer KC, et al. A new classification system for chronic lung allograft dysfunction. J Heart Lung Transplant 2014;33:127-33.

3. Verleden GM, Glanville AR, Lease ED, et al. Chronic lung allograft dysfunction: definition, diagnostic criteria, and approaches to treatment-A consensus report from the Pulmonary Council of the ISHLT. J Heart Lung Transplant 2019;38:493-503.

4. Sato M, Waddell TK, Wagnetz U, et al. Restrictive allograft syndrome (RAS): a novel form of chronic lung allograft dysfunction. J Heart Lung Transplant 2011;30:735-42.

5. Glanville AR, Verleden GM, Todd JL, et al. Chronic lung allograft dysfunction: Definition and update of restrictive allograft syndrome. A consensus report from the Pulmonary Council of the ISHLT. J Heart Lung Transplant 2019;38:483-92.

6. Levy L, Huszti E, Renaud-Picard B, et al. Risk assessment of chronic lung allograft dysfunction phenotypes: Validation and proposed refinement of the 2019 International Society for Heart and Lung Transplantation classification system. J Heart Lung Transplant 2020;39:761-70.

7. Jungraithmayr W, Jang JH, Schrepfer S, et al. Small Animal Models of Experimental Obliterative Bronchiolitis. Am J Respir Cell Mol Biol 2013;48:675-84.

8. Atanasova S, Hirschburger M, Jonigk D, et al. A relevant experimental model for human bronchiolitis obliterans syndrome. J Heart Lung Transplant 2013;32:1131-9.

9. Krenn K, Gmeiner M, Paulus P, et al. Effects of azithromycin and tanomastat on experimental bronchiolitis obliterans. J Thorac Cardiovasc Surg 2015;149:1194-202.

10. Lunardi F, Zampieri D, Vadori M, et al. Overexpression of hypoxia-inducible factor- $1 \alpha$ in primary graft dysfunction developing in an orthotopic lung transplantation rat model. Transplant Proc 2017;49:722-5.

11. Zampieri D, Azzollini N, Vuljan S, et al. Vein suturing results in worse lung graft outcomes compared to the cuff method. Eur Surg Res 2019;60:106-16.

12. Stewart S, Fishbein MC, Snell GI, et al. Revision of the 1996 working formulation for the standardization of nomenclature in the diagnosis of lung rejection. J Heart Lung Transplant 2007;26:1229-42. 
13. Martinu T, Oishi H, Juvet SC, et al. Spectrum of chronic lung allograft pathology in a mouse minor-mismatched orthotopic lung transplant model. Am J Transplant 2019;19:247-58.

14. R Core Team. R: A language and environment for statistical computing. Vienna: R Foundation for statistical computing, 2019.

15. Markopoulo KD, Cool CD, Elliot TL, et al. Obliterative bronchiolitis: varying presentations and clinicopathological correlation. Eur Respir J 2002;19:20-30.

16. Martinu T, Howell DN, Davis RD, et al. Pathologic correlates of bronchiolitis obliterans syndrome in pulmonary retransplant recipients. Chest 2006;129:1016-23.

17. Boehler A, Estenne M. Post-transplant bronchiolitis obliterans. Eur Respir J 2003;22:1007-18.

18. Reichenspurner H, Girgis RE, Robbins RC, et al. Stanford experience with obliterative bronchiolitis after lung and heart-lung transplantation. Ann Thorac Surg 1996;62:1467-72; discussion 1472-3.

19. Verleden SE, Vasilescu DM, McDonough JE, et al. Linking clinical phenotypes of chronic lung allograft dysfunction to changes in lung structure. Eur Respir J 2015;46:1430-9

20. Husain AN, Garrity ER. Lung Transplantation. The state of the airways. Arch Pathol Lab Med 2016;140:241-4.

21. Ofek E, Sato M, Saito T, et al. Restrictive allograft syndrome post lung transplantation is characterized by pleuroparenchymal fibroelastosis. Mod Pathol 2013;26:350-6.

22. Montero MA, Osadolor T, Khiroya R, et al. Restrictive allograft syndrome and idiopathic pleuroparenchymal fibroelastosis: do they really have the same histology? Histopathology 2017;70:1107-13.

23. von der Thüsen JH, Vandermeulen E, Vos R, et al. The histomorphological spectrum of restrictive chronic lung allograft dysfunction and implications for prognosis. Mod Pathol 2018;31:780-90.

24. Todd, JL, Jain, R, Pavlisko, EN et al. Impact of forced vital capacity loss on survival after the onset of chronic lung allograft dysfunction. Am J Respir Crit Care Med 2014;189:159-66

25. Roux A, Bendib L, Holifanjaniaina S, et al. AntibodyMediated Rejection in lung transplantation: clinical outcomes and donor-specific antibody characteristics. Am J Transplant 2016;16:1216-28.

26. Verleden SE, Vanaudenaerde BM, Emonds MP, et al. Donor-specific and -nonspecific HLA antibodies and outcome post lung transplantation. Eur Respir J
2017;50:1701248.

27. Walton DC, Hiho SJ, Cantwell LS, et al. HLA Matching at the eplet level protects against chronic lung allograft dysfunction. Am J Transplant 2016;16:2695-703.

28. Costa AN, Carraro RM, Nascimento EC, et al. Acute fibrinoid organizing pneumonia in lung transplant the most feared allograft dysfunction. Transplantation 2016;100:e11-2.

29. Watanabe T, Martinu T, Chruscinski A, et al. A B celldependent pathway drives chronic lung allograft rejection after ischemia-reperfusion injury in mice. Am J Transplant 2019;19:3377-89.

30. Sato M, Hirayama S, Hwang DM, et al. The role of intrapulmonary de novo lymphoid tissue in obliterative bronchiolitis after lung transplantation. J Immunol 2009;182:7307-16.

31. Sato M, Hirayama S, Matsuda Y, et al. Stromal activation and formation of lymphoid-like stroma in chronic lung allograft dysfunction. Transplantation 2011;91:1398-405.

32. Vandermeulen E, Lammertyn E, Verleden SE, et al. Immunological diversity in phenotypes of chronic lung allograft dysfunction: a comprehensive immunohistochemical analysis. Transpl Int 2017;30:134-43.

33. Verleden SE, Ruttens D, Vandermeulen E, et al. Predictors of survival in restrictive chronic lung allograft dysfunction after lung transplantation. J Heart Lung Transplant 2016;35:1078-84.

34. Snyder LD, Wang Z, Chen DF, et al. Implications for human leukocyte antigen antibodies after lung transplantation: a 10-year experience in 441 patients. Chest 2013;144:226-33.

35. Ju L, Suberbielle C, Li X, et al. HLA and lung transplantation. Front Med 2019;13:298-313.

36. Lin X, Li W, Lai J, et al. Five-year update on the mouse model of orthotopic lung transplantation: Scientific uses, tricks of the trade, and tips for success. J Thorac Dis 2012;4:247-58.

37. Seetharam A, Tiriveedhi V, Mohanakumar T. Alloimmunity and autoimmunity in chronic rejection. Curr Opin Organ Transplant 2010;15:531-6.

Cite this article as: Pezzuto F, Lunardi F, Vadori M, Zampieri D, Casiraghi F, Azzollini N, Vuljan SE, Mammana M, Vedovelli L, Schiavon M, Gregori D, Cozzi E, Rea F, Calabrese F. Chronic lung allograft pathology lesions in two rat strain combinations. J Thorac Dis 2021;13(5):2833-2843. doi: 10.21037/ jtd-20-3415 